\title{
ON NEW FRACTAL PHENOMENA CONNECTED WITH INFINITE LINEAR IFS
}

\author{
SERGIO ALBEVERIO ${ }^{1,2,3,4}$, YURI KONDRATIEV ${ }^{6,7,9}$ \\ ROMAN NIKIFOROV ${ }^{8}$, GRYGORIY TORBIN $^{9,10}$
}

\begin{abstract}
We establish several new fractal and number theoretical phenomena connected with expansions which are generated by infinite linear iterated function systems. First of all we show that the systems $\Phi$ of cylinders of generalized Lüroth expansions are, generally speaking, not faithful for the Hausdorff dimension calculation. Using Yuval Peres' approach, we prove sufficient conditions for the non-faithfulness of such families of cylinders. On the other hand, rather general sufficient conditions for the faithfulness of such covering systems are also found. As a corollary of our main results, we obtain the non-faithfullness of the family of cylinders generated by the classical Lüroth expansion.

Possible infinite entropy of the stochastic vector $Q_{\infty}$ which determines the metric relations for partitions of the generalized Lüroth expansions, possible non-faithfulness of the family $\Phi\left(Q_{\infty}\right)$ and the absence of general formulae for the calculation of the Hausdorff dimension even for probability measures with independent identically distributed symbols of generalized Lüroth expansions are all facts that do not allow to apply usual probabilistic methods, as well as methods of dynamical systems to study fractal properties of corresponding subsets of non-normal numbers. Since the 'divergent points technique' is not developed for measures generated by infinite IFS, the corresponding methods are also not applicable to solve the problem of the above fractal properties. Despite of the above mentioned difficulties, we develop new approach to the study of subsets of $Q_{\infty^{-}}$ essentially non-normal numbers and prove (without any additional restrictions on the stochastic vector $Q_{\infty}$ ) that this set is superfractal. This result answers the open problem mentioned in 2] and completes the metric, dimensional and topological classification of real numbers via the asymptotic behaviour of frequencies their digits in the generalized Lüroth expansion.
\end{abstract}

${ }^{1}$ Institut für Angewandte Mathematik, Universität Bonn, Endenicher Allee 60, D-53115 Bonn (Germany); ${ }^{2} \mathrm{HCM}$ and IZKS, Bonn; ${ }^{3}$ BiBoS, Bielefeld-Bonn; ${ }^{4}$ CERFIM, Locarno; E-mail: albeverio@iam.uni-bonn.de

${ }^{6}$ Fakultät für Mathematik, Universität Bielefeld, Postfach 1001 31, D-33501, Bielefeld (Germany) ${ }^{7}$ BiBoS, Bielefeld-Bonn; E-mail: kondrat@mathematik.uni-bielefeld.de

8 National Pedagogical University, Pyrogova str. 9, 01030 Kyiv (Ukraine); E-mail: rnikiforov@gmail.com

${ }^{9}$ National Pedagogical University, Pyrogova str. 9, 01030 Kyiv (Ukraine) ${ }^{10}$ Institute for Mathematics of NASU, Tereshchenkivs'ka str. 3, 01601 Kyiv (Ukraine); E-mail: torbin@iam.uni-bonn.de (corresponding author)

AMS Subject Classifications (2010): 11K55, 28A80, 60G30.

Key words: infinite IFS, Lüroth expansion, $Q_{\infty}$-expansion, Hausdorff dimension, faithful and non-faithful nets, fractals, singular probability measures, non-normal numbers.

\section{INTRODUCTION}

There is a rich diversity of systems of numerations for real numbers (see, e. g., [19, 45] and references therein). Any such system generates its own metric, dimensional and probabilistic 
theories. A very important family of such systems consists of expansions generated by iterated function systems (IFS) and admit a dynamical approach to their treatment ([19, 39, 45]). For the case of finite IFS, fractal properties of corresponding attractors and invariant measures were intensively studied during the last 50 years. In particular, it has been shown that methods of the ergodic theory of dynamical systems and statistical physics are of great importance to study fine fractal properties of related sets and measures (see, e. g., Bowen [17], Ruelle [44], Falconer [22] and references therein). An important part of the above mentioned studies is related to the distribution of frequencies digits in expansions for points in the invariant sets of IFS (see, e. g., [9, 10, 12, 14, 20, 34, 35, 40, 50,).

The corresponding theory for infinite IFS is essentially less developed and a lot of new phenomena appear in such a case. It is known, for instance, that even for the infinite linear IFS the Hausdorff dimension of the corresponding attractor fails to be the root of the equation $\sum_{i=1}^{\infty} k_{i}^{x}=1$, where $k_{i}$ is the similarity ratio of $F_{i}$ (see, e.g., [32] and references therein).

The most famous expansions with infinite alphabets are the classical continued fractions expansion and the Lüroth expansion $([19,45])$. Problems of the dimensional theory of continued fraction expansion are well known (see, e. g., [24, 25, 26, 27, 28, 29, 30]) and can partially be explained by the new phenomenon of fractal non-faithfulness in the dimensional theory of continued fraction expansions which has been discovered recently in [38. In the present paper we deal with expansions generated by infinite linear IFS $\left\{F_{0}, F_{1}, \ldots, F_{n}, \ldots\right\}$ such that $F_{n}$ is a similarity transformation with ratio $q_{n}, \sum_{n=0}^{\infty} q_{n}=1$ and the sequence $\left\{\sup F_{i}([0,1])\right\}$ is strictly monotone. Let

$$
\Delta_{\alpha_{1}(x) \alpha_{2}(x) \ldots \alpha_{n}(x) \ldots}
$$

be the corresponding expansion of $x \in[0,1]$. Let us mention that if the above sequence $\left\{\sup F_{i}([0,1])\right\}$ is strictly decreasing and $q_{i}=\frac{1}{(i+1)(i+2)}$, then we get the classical Lüroth expansion. For the case of a increasing sequence we get the $Q_{\infty}$-expansion. Since metric, dimensional and probabilistic theories are entirely the same for both cases, we will consider only the case where the above sequence increases. Such an expansion is actually the $f$ expansion (see, e.g., [21, 42] for details), which is generated by the following strictly increasing continuous function $f$ defined on $[0,+\infty)$ such that $f(0)=0$ and $f$ increases linearly on each interval $[i, i+1]$ with $f(i+1)-f(i)=q_{i}, \forall i \in \mathbb{N}_{0}$.

One approach to the simplification of the calculation of the Hausdorff dimension consists in some restrictions of admissible coverings. This idea came from Besicovitch's works and has been used by Rogers and Taylor to construct comparable net measures ([43]) as approximations of the Hausdorff measures. In this paper we develop Besicovitch's approach via construction of net coverings which lead to a special family of net measures which are more general that comparable ones. The first phenomenon we will talk about is connected with the problem of faithfulness and non-faithfulness of the family of cylinders from the above expansions for the Hausdorff dimension calculation. To be precise, let us shortly recall that the $\alpha$-dimensional Hausdorff measure of a set $E \subset[0,1]$ with respect to a given family of coverings $\Phi$ is defined by

$$
H^{\alpha}(E, \Phi)=\lim _{\epsilon \rightarrow 0} \inf _{\left|E_{j}\right| \leq \epsilon} \sum_{j}\left|E_{j}\right|^{\alpha}=\lim _{\epsilon \rightarrow 0} H_{\epsilon}^{\alpha}(E, \Phi)
$$


where the infimum is taken over all at most countable $\epsilon$-coverings $\left\{E_{j}\right\}$ of $E, E_{j} \in \Phi$. The nonnegative number

$$
\operatorname{dim}_{H}(E, \Phi)=\inf \left\{\alpha: H^{\alpha}(E, \Phi)=0\right\}
$$

is called the Hausdorff dimension of the set $E \subset[0,1]$ w.r.t. the family $\Phi$. If $\Phi$ is the family of all subsets of $[0,1]$, or $\Phi$ coincides with the family of all closed (open) subintervals of

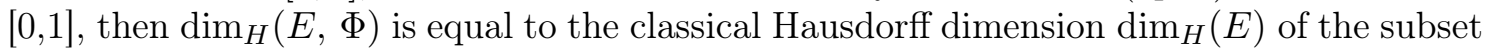
$E \subset[0,1]$.

A fine covering family $\Phi$ is said to be a faithful family of coverings (non-faithful family of coverings) for the Hausdorff dimension calculation on $[0,1]$ if

$$
\begin{gathered}
\operatorname{dim}_{H}(E, \Phi)=\operatorname{dim}_{H}(E), \quad \forall E \subseteq[0,1] \\
\text { (resp. } \left.\exists E \subseteq[0,1]: \operatorname{dim}_{H}(E, \Phi) \neq \operatorname{dim}_{H}(E)\right) .
\end{gathered}
$$

It is clear that any family $\Phi$ of comparable net-coverings (i. e., net-coverings which generate comparable net-measures) is faithful. Conditions for Vitali coverings to be faithful were studied by many authors (see, e.g., [3, 15, 18, 41] and references therein). First steps in this direction have been done by A. Besicovitch ([14]), who proved the faithfulness for the family of cylinders of a binary expansion. His result was extended by P. Billingsley ([15]) to the family of $s$-adic cylinders, by M. Pratsiovytyi ([49]) to the family of $Q-S$ cylinders, and by S. Albeverio and G. Torbin ([3]) to the family of $Q^{*}$-cylinders for those matrices $Q^{*}$ whose elements $p_{0 k}, p_{(s-1) k}$ are bounded away from zero.

It is rather paradoxical that initial examples of non-faithful families of coverings appeared firstly in the two-dimensional case (as a result of active studies of self-affine sets during the last decade of XX century (see, e.g., [13])). The family of cylinders of the classical continued fraction expansion can probably be considered as the first (and rather unexpected) example of non-faithful one-dimensional net-family of coverings ([38]). It is clear that for the family of all cylinders of the $Q_{\infty}$-expansion neither assumptions for a Vitali covering ([18]) nor any other known conditions for the faithfulness do hold. By using the approach invented by Yuval Peres to prove the non-faithfulness of the family of continued fraction cylinders ([38]), in Section 2 of the present paper we prove the non-faithfulness for the family $\Phi\left(Q_{\infty}\right)$ of cylinders of the $Q_{\infty}$-expansion with polynomially decreasing elements $\left\{q_{i}\right\}$ (Theorem 2), which shows that the family of cylinders of the Lüroth expansion is non-faithful! On the other hand we give rather general sufficient conditions for $\Phi\left(Q_{\infty}\right)$ to be faithful (Theorem 1). Let us stress that this family of expansions is the first known one generating faithful as well as non-faithful nets.

The second aim of the paper is to study properties of the set of $Q_{\infty}$-essentially nonnormal numbers and obtain a complete metric, topological and fractal classification of real numbers via the asymptotic behaviour of frequencies of their $Q_{\infty}$-digits. To be more precise, let $N_{i}(x, n)$ be the number of digits $i$ among the first $n$ digits of the $Q_{\infty}$-expansion of $x$. If the limit $\lim _{n \rightarrow \infty} \frac{N_{i}(x, n)}{n}$ exists, then its value $\nu_{i}(x)$ is said to be the asymptotic frequency of the digit ' $i$ ' in the $Q_{\infty}$-expansion of $x$. By the law of large numbers, for Lebesgue almost all real numbers from the unit interval we have $\nu_{i}(x)=q_{i}, \forall i \in \mathbb{N}_{0}$.

The set $N\left(Q_{\infty}\right)=\left\{x: \forall i \in \mathbb{N}_{0}, \lim _{n \rightarrow \infty} \frac{N_{i}(x, n)}{n}=q_{i}\right\}$ is said to be the set of $Q_{\infty}$-normal numbers.

The set $W\left(Q_{\infty}\right)=\left\{x: \forall i \in \mathbb{N}_{0}, \lim _{n \rightarrow \infty} \frac{N_{i}(x, n)}{n}\right.$ exists $\} \cap \overline{N\left(Q_{\infty}\right)}$ is said to be the set of $Q_{\infty}$-quasi-normal numbers, where $\frac{{ }^{\prime \rightarrow \infty}}{N\left(Q_{\infty}\right)}=[0,1) \backslash N\left(Q_{\infty}\right)$. 
The set $D\left(Q_{\infty}\right)=\left\{x: \exists i_{0} \in N_{0}, \liminf _{n \rightarrow \infty} \frac{N_{i_{0}}(x, n)}{n}<\limsup _{n \rightarrow \infty} \frac{N_{i_{0}}(x, n)}{n}\right\}$ is said to be the set of $Q_{\infty}$-non-normal numbers.

The set

$$
\begin{aligned}
& P\left(Q_{\infty}\right)=\left\{x: \quad \exists i_{0} \in \mathbb{N}_{0}, \liminf _{n \rightarrow \infty} \frac{N_{i_{0}}(x, n)}{n}<\limsup _{n \rightarrow \infty} \frac{N_{i_{0}}(x, n)}{n},\right. \\
& \left.\exists i_{1} \in \mathbb{N}_{0}, \liminf _{n \rightarrow \infty} \frac{N_{i_{1}}(x, n)}{n}=\limsup _{n \rightarrow \infty} \frac{N_{i_{1}}(x, n)}{n}\right\}
\end{aligned}
$$

is said to be the set of $Q_{\infty}$-partially non-normal numbers.

The set $L\left(Q_{\infty}\right)=\left\{x: \forall i \in \mathbb{N}_{0}, \liminf _{n \rightarrow \infty} \frac{N_{i}(x, n)}{n}<\limsup _{n \rightarrow \infty} \frac{N_{i}(x, n)}{n}\right\}$ is said to be the set of $Q_{\infty}$-essentially non-normal numbers.

For the case of the $s$-adic expansion $(s>1, s \in \mathbb{N})$ and some generalizations for expansions with finite alphabets several approaches have been developed to study properties of the corresponding sets. In 1995 it has been shown ([40]) that the set of non-normal numbers w.r.t. base $s$ is superfractal (i. e., a set of zero Lebesgue measure and of full Hausdorff dimension). By using different approaches, in [9] and in [12] it has been proven that the set of real numbers which are essentially non-normal w.r.t. base $s$ is also superfractal. Another powerful approach (the so-called 'divergence points' approach) was developed in a series of papers by L. Olsen, S. Winter, N. Snigereva, I. S. Baek, A. Bisbas (see, e.g., [10, 16, 34, 35, 36, 37] and references therein). From Volkmann results [50] it follows that this set is residual, and, therefore, other three subsets $N\left(Q_{\infty}\right), W\left(Q_{\infty}\right), P\left(Q_{\infty}\right)$ are of the first Baire category.

For the case of infinite IFS the situation is essentially more complicated. In [23] the authors pointed out that "the difference from earlier works is the (countable) infinity of the alphabet, from which comes a particular phenomenon that the formal variational principle does not hold as in the case of compact dynamics and ... the thermodynamic formalism does not work as one wishes. In fact, we do not know whether there always exist a Gibbs measure, to be suitable defined, on the set in question, which has the dimension of the set." In the same paper [23] the authors also generalized results from [11] and studied fractal properties of Besicovitch-Eggleston sets, which are subsets of $W\left(Q_{\infty}\right)$, found explicit formulae for their Hausdorff dimension and stressed significant differences from those in the $s$-adic expansion.

Possible infinite entropy of the stochastic vector $Q_{\infty}$, possible non-faithfulness of the family $\Phi\left(Q_{\infty}\right)$ and the absence of general formulae for the calculation of the Hausdorff dimension even for probability measures with independent identically distributed $Q_{\infty^{-}}$ symbols (this is still an open problem (see, e.g., [11, 32, 33])) do not allow us to apply methods from [9] to study fractal properties of the above defined sets. Let us also mention that methods from [34, 35, 37] are also not applicable to solve the problem because of the absence of 'divergent points techniques' for the measures generated by infinite IFS.

Despite the above mentioned difficulties, in the last section of the present work we develop a new approach to the study of subsets of $Q_{\infty}$-essentially non-normal numbers and prove (without any additional restrictions on the stochastic vector $Q_{\infty}$ like convergence of the series $\sum_{j=0}^{\infty} \frac{\ln ^{2} q_{j}}{2^{j}}$ (see, e.g., [2]), finiteness of the entropy or faithfulness of the corresponding family $\left.\Phi\left(Q_{\infty}\right)\right)$ that this set is superfractal. This result answers the open problem mentioned in 2] and completes the metric, dimensional and topological classification of 
real numbers via the asymptotic behaviour of frequencies of their digits in the generalized Lüroth expansion:

\begin{tabular}{|c|c|c|c|}
\hline & Lebesgue measure & Hausdorff dimension & Baire category \\
\hline$N\left(Q_{\infty}\right)$ & 1 & 1 & first \\
\hline$W\left(Q_{\infty}\right)$ & 0 & 1 & first \\
\hline$P\left(Q_{\infty}\right)$ & 0 & 1 & first \\
\hline$L\left(Q_{\infty}\right)$ & 0 & 1 & second \\
\hline
\end{tabular}

\section{On FAITHFul AND NON-FAithFul COVERING SYSTEMS GENERATED BY $Q_{\infty}$-EXPANSION}

In this section we shall demonstrate that fractal non-faithful phenomena for one-dimensional nets can appear even for those nets which are generated by linear infinite IFS. Non-faithfulness for the Hausdorff dimension calculation of the family of cylinders for the classical Lüroth expansion is a direct consequence of our results.

For the convenience of the reader we give a geometrical explanation of the above mentioned $Q_{\infty}$-expansion. Given a $Q_{\infty}$-vector we consecutively perform decompositions of the semi-interval $[0,1)$ in the following way.

Step 1. We decompose $[0,1)$ (from the left to the right) into the union of semi-intervals $\Delta_{\alpha_{1}}, \alpha_{1} \in \mathbb{N}_{0}$ (without common points) of the length $\left|\Delta_{\alpha_{1}}\right|=q_{\alpha_{1}}$,

$$
[0,1)=\bigcup_{\alpha_{1}=0}^{\infty} \Delta_{\alpha_{1}}
$$

Each interval $\Delta_{\alpha_{1}}$ is called a 1-rank cylinder (basic interval).

Step $n \geq 2$. We decompose (from the left to the right) each $(n-1)$-rank cylinder $\Delta_{\alpha_{1} \ldots \alpha_{n-1}}$ into the union of $n$-rank cylinders $\Delta_{\alpha_{1} \ldots \alpha_{n}}$ (without common points)

$$
\Delta_{\alpha_{1} \ldots \alpha_{n-1}}=\bigcup_{\alpha_{n}=0}^{\infty} \Delta_{\alpha_{1} \ldots \alpha_{n}},
$$

whose lengths

$$
\left|\Delta_{\alpha_{1} \alpha_{2} \ldots \alpha_{n}}\right|=q_{\alpha_{1}} \cdot q_{\alpha_{2}} \cdots q_{\alpha_{n}}=\prod_{i=1}^{n} q_{\alpha_{i}}
$$

are related as follows

$$
\left|\Delta_{\alpha_{1} \ldots \alpha_{n-1} 0}\right|:\left|\Delta_{\alpha_{1} \ldots \alpha_{n-1} 1}\right|: \cdots:\left|\Delta_{\alpha_{1} \ldots \alpha_{n-1} \alpha_{n}}\right|: \cdots=q_{0}: q_{1}: \cdots: q_{\alpha_{n}}: \cdots .
$$

It is clear that any sequence of indices $\left\{\alpha_{n}\right\}$ generates the corresponding sequence of embedded cylinders

$$
\Delta_{\alpha_{1}} \supset \Delta_{\alpha_{1} \alpha_{2}} \supset \cdots \supset \Delta_{\alpha_{1} \alpha_{2} \ldots \alpha_{n}} \supset \cdots
$$

and there exists a unique point $x \in[0,1)$ belonging to all of them.

Conversely, for any point $x \in[0,1)$ there exists a unique sequence of embedded cylinders $\Delta_{\alpha_{1}} \supset \Delta_{\alpha_{1} \alpha_{2}} \supset \ldots \supset \Delta_{\alpha_{1} \alpha_{2} \ldots \alpha_{n}} \supset \ldots$ containing $x$, i. e.,

$$
\begin{gathered}
x=\bigcap_{n=1}^{\infty} \Delta_{\alpha_{1} \ldots \alpha_{n}}=\bigcap_{n=1}^{\infty} \Delta_{\alpha_{1}(x) \ldots \alpha_{n}(x)}=: \Delta_{\alpha_{1}(x) \ldots \alpha_{n}(x) \ldots ;} ; \\
x=\Delta_{\alpha_{1}(x) \ldots \alpha_{n}(x) \ldots .}
\end{gathered}
$$

The expression is said to be the $Q_{\infty}$-expansion for $x$. Real numbers which are end-points of n-th rank cylinders are said to be $Q_{\infty}$-rational, and their $Q_{\infty}$-expansion contains only 
finitely many non-zero digits. In the sequel, $\Phi=\Phi\left(Q_{\infty}\right)$ will be the family of all possible cylinders of the $Q_{\infty}$-partition of the semi-interval $[0,1)$, i. e.,

$$
\Phi=\left\{E: E=\Delta_{\alpha_{1} \ldots \alpha_{n}}, \quad \alpha_{i} \in \mathbb{N}_{0}, i=1,2, \ldots, n ; \quad n \in \mathbb{N}\right\} .
$$

Before we present the new phenomena related to the non-faithfulness of the nets generated by infinite IFS, we give rather general sufficient conditions for nets $\Phi$ generated by the $Q_{\infty}$-expansion to be faithful.

Theorem 1. Let $Q_{\infty}=\left(q_{0}, \ldots, q_{i}, \ldots\right)$ be a stochastic vector such that for any $\alpha>0$ there exists a constant $c=c(\alpha)$ with

$$
\sum_{k=i+1}^{\infty} q_{k}^{\alpha} \leq c(\alpha) q_{i}^{\alpha}, \forall i \in \mathbb{N}_{0}
$$

Then the family $\Phi$ is faithful for the Hausdorff dimension calculation on the unit interval.

Proof. For the calculation of the Hausdorff dimension of a set $E \subset[0,1)$ we may consider only coverings of intervals $\left[a_{j}, b_{j}\right)$ such that $a_{j} \in A, b_{j} \in A$ and the set $A$ is an everywhere dense set on $[0,1]$. Let us choose $A$ to be the set of all $Q_{\infty}$-rational numbers.

For a given set $E, \alpha>0, \epsilon>0$ let us choose $\delta \in(0, \alpha)$, and let $\left\{E_{j}\right\}$ be an $\epsilon$-covering of $E$ by above intervals $E_{j}=\left[a_{j}, b_{j}\right), a_{j} \in A, b_{j} \in A$.

For any $j \in \mathbb{N}$ there exists the cylinder $\Delta_{\alpha_{1} \alpha_{2} \ldots \alpha_{n_{j}}}$ of maximal rank that contains $\left[a_{j}, b_{j}\right)$ (i. e., any cylinder of $\left(n_{j}+1\right)$-th rank does not contain $\left.\left[a_{j}, b_{j}\right)\right)$. So, the $a_{j}$ have the following $Q_{\infty}$-expansion:

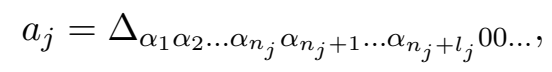

where $\alpha_{k}=\alpha_{k}\left(a_{j}\right)$. Then, the point $c_{j}=\Delta_{\alpha_{1} \ldots \alpha_{n_{j}}\left(\alpha_{n_{j}+1}+1\right) 00 \ldots}$ belongs to $\left[a_{j}, b_{j}\right)$.

Let $\beta_{k}:=\alpha_{n_{j}+k}\left(a_{j}\right)$ be the $\left(n_{j}+k\right)$-th digit of the $Q_{\infty}$-expansion of $a_{j}, k \in\left\{1,2, \ldots, l_{j}\right\}$. To cover $E_{j}$ by cylinders we consider the coverings of $\left[a_{j}, c_{j}\right)$ and $\left[c_{j}, b_{j}\right)$ separately.

If the cylinder $\Delta_{\alpha_{1} \ldots \alpha_{n_{j}}\left(\beta_{1}+1\right)}$ belongs to $\left[c_{j}, b_{j}\right)$, then $\left[c_{j}, b_{j}\right)$ can be covered by cylinders

$$
\Delta_{\alpha_{1} \ldots \alpha_{n_{j}}\left(\beta_{1}+i\right)}, i \in \mathbb{N} .
$$

The $\alpha$-volume of this covering is equal to

$$
\begin{aligned}
\sum_{i=1}^{\infty}\left|\Delta_{\alpha_{1} \ldots \alpha_{n_{j}}\left(\beta_{1}+i\right)}\right|^{\alpha} \leq & \left|\Delta_{\alpha_{1} \ldots \alpha_{n_{j}}\left(\beta_{1}+1\right)}\right|^{\alpha}+\sum_{i=2}^{\infty}\left|\Delta_{\alpha_{1} \ldots \alpha_{n_{j}}\left(\beta_{1}+i\right)}\right|^{\alpha} \leq \\
& \leq(1+c)\left|\Delta_{\alpha_{1} \ldots \alpha_{n_{j}}\left(\beta_{1}+1\right)}\right|^{\alpha} \leq(1+c)\left|E_{j}\right|^{\alpha} .
\end{aligned}
$$

If the cylinder $\Delta_{\alpha_{1} \ldots \alpha_{n_{j}}\left(\beta_{1}+1\right)}$ does not belong to $\left[c_{j}, b_{j}\right)$, then there exists a positive integer $t_{j}$ such that the cylinder

$$
\Delta_{\alpha_{1} \ldots \alpha_{n_{j}}\left(\beta_{1}+1\right)} \underbrace{0 \ldots 0}_{t_{j}}
$$

contains $\left[c_{j}, b_{j}\right)$ and the cylinder $\Delta_{\alpha_{1} \ldots \alpha_{n_{j}}}\left(\beta_{1}+1\right) \underbrace{0 \ldots 0}_{t_{j}+1}$ is contained in $\left[c_{j}, b_{j}\right)$.

In such a case the cylinder (2) covers $\left[c_{j}, b_{j}\right)$ and its diameter does not exceed $\frac{1}{q_{0}}\left|E_{j}\right|$. So, $\left[c_{j}, b_{j}\right)$ can be covered by cylinders from $\Phi$ such that the corresponding $\alpha$-volume does not exceed $B_{0}^{\alpha}\left|E_{j}\right|^{\alpha}$, where $B_{0}=\max \left\{1+c, \frac{1}{q_{0}}\right\}$.

The most difficult point in the proof is to find small enough (in the sense of $\alpha$-volume) covering of $\left[a_{j}, c_{j}\right)$ by cylinders from $\Phi$. 
The set $\left[a_{j}, c_{j}\right)$ contains non-intersecting cylinders of the following ranks.

$$
\begin{aligned}
\operatorname{Rank}\left(n_{j}+2\right): & \Delta_{\alpha_{1} \ldots \alpha_{n_{j}} \beta_{1} i}, \quad i>\beta_{2} . \\
& \vdots \\
\operatorname{Rank}\left(n_{j}+l_{j}-1\right): & \Delta_{\alpha_{1} \ldots \alpha_{n_{j}} \beta_{1} \ldots \beta_{l_{j}-2} i}, \quad i>\beta_{l_{j}-1} . \\
\operatorname{Rank}\left(n_{j}+l_{j}\right): & \Delta_{\alpha_{1} \ldots \alpha_{n_{j}} \beta_{1} \ldots \beta_{l_{j}-1} i}, \quad i \geq \beta_{l_{j}} .
\end{aligned}
$$

We recall that $a_{j}=\inf \Delta_{\alpha_{1} \ldots \alpha_{n_{j}} \beta_{1} \beta_{2} \ldots \beta_{l_{j}-1} \beta_{l_{j}}}$.

So, $\left[a_{j}, c_{j}\right)$ is split into the union of the above cylinders of ranks $\left(n_{j}+2\right), \ldots,\left(n_{j}+l_{j}\right)$. The $\alpha$-volume of cylinders of $\left(n_{j}+k\right)$-th rank $\left(k<l_{j}\right)$ is equal to

$$
\sum_{i=\beta_{k}+1}^{\infty}\left|\Delta_{\alpha_{1} \ldots \alpha_{n_{j}} \beta_{1} \ldots \beta_{k-1} i}\right|^{\alpha} \leq(1+c)\left|\Delta_{\alpha_{1} \ldots \alpha_{n_{j}} \beta_{1} \ldots \beta_{k-1}\left(\beta_{k}+1\right)}\right|^{\alpha}
$$

and

$$
\sum_{i=\beta_{l_{j}}}^{\infty}\left|\Delta_{\alpha_{1} \ldots \alpha_{n_{j}} \beta_{1} \ldots \beta_{l_{j}-1} i}\right|^{\alpha} \leq(1+c)\left|\Delta_{\alpha_{1} \ldots \alpha_{n_{j}} \beta_{1} \ldots \beta_{l_{j}-1} \beta_{l_{j}}}\right|^{\alpha} .
$$

Thus $\left[a_{j}, c_{j}\right)$ can be covered by a countable number of above cylinders, and the corresponding $\alpha$-volume does not exceed the value

$$
\mathrm{v}_{j}(\alpha)=(1+c)\left(\left|\Delta_{\alpha_{1} \ldots \alpha_{n_{j}}\left(\beta_{1}+1\right)}\right|^{\alpha}+\cdots+\left|\Delta_{\alpha_{1} \ldots \alpha_{n_{j}} \beta_{1} \ldots \beta_{l_{j}}}\right|^{\alpha}\right) .
$$

Unfortunately in the latter sum the distribution of lengths of cylinders can be arbitrary. In particular, even the cylinder of maximal rank can be of maximal length (one can check this by considering the case where $\left.q_{i}=\frac{a}{2^{2^{i}}}\right)$, and the sequence $\left\{l_{j}\right\}$ is, generally speaking, unbounded.

For a given $l_{j} \in \mathbb{N}$ one can choose $d_{j} \in \mathbb{N}$ such that $2^{d_{j}-1}<l_{j} \leq 2^{d_{j}}$. Let $\mathbf{q}:=\max _{i}\left(q_{i}\right)$. Then the above sum (3) can be rewritten as follows:

$$
\begin{aligned}
\mathrm{v}_{j}(\alpha) & =(1+c)\left[\sum_{i=1}^{d_{j}-1} \sum_{2^{i-1}<k \leq 2^{i}}\left|\Delta_{\alpha_{1} \ldots \alpha_{n_{j}} \beta_{1} \ldots \beta_{k-1}\left(\beta_{k}+1\right)}\right|^{\alpha}+\right. \\
& \left.+\sum_{2^{d_{j}-1}<k \leq l_{j}-1}\left|\Delta_{\alpha_{1} \ldots \alpha_{n_{j}} \beta_{1} \ldots \beta_{k-1}\left(\beta_{k}+1\right)}\right|^{\alpha}+\left|\Delta_{\alpha_{1} \ldots \alpha_{n_{j}} \beta_{1} \ldots \beta_{l_{j}}}\right|^{\alpha}\right] \leq \\
& \leq(1+c)\left|E_{j}\right|^{\alpha-\delta} \sum_{k=0}^{d_{j}-1} 2^{k}\left(\mathbf{q}^{\delta}\right)^{2^{k}}<(1+c)\left|E_{j}\right|^{\alpha-\delta} \sum_{s=1}^{\infty} s\left(\mathbf{q}^{\delta}\right)^{\frac{s}{2}}\left(\mathbf{q}^{\delta}\right)^{\frac{s}{2}}
\end{aligned}
$$

for any $\delta \in(0, \alpha)$.

It is clear that there exists a constant $W(\delta)$ such that $s\left(\mathbf{q}^{\delta}\right)^{\frac{s}{2}} \leq W(\delta), \forall s \in \mathbb{N}$. Therefore,

$$
\mathrm{v}_{j}(\alpha) \leq(1+c)\left|E_{j}\right|^{\alpha-\delta} W(\delta) \sum_{s=1}^{\infty}\left(\mathbf{q}^{\delta}\right)^{\frac{s}{2}}=\frac{(1+c) W(\delta) \mathbf{q}^{\frac{\delta}{2}}}{1-\mathbf{q}^{\frac{\delta}{2}}}\left|E_{j}\right|^{\alpha-\delta}
$$


So, for a given $E_{j}=\left[a_{j}, b_{j}\right)$ there exists a countable family of cylinders that cover $E_{j}$ and whose $\alpha$-volume does not exceed $K(\alpha, \delta)\left|E_{j}\right|^{\alpha-\delta}$ with

$$
K(\alpha, \delta):=B_{0}+\frac{(1+c(\alpha)) W(\delta) \mathbf{q}^{\frac{\delta}{2}}}{1-\mathbf{q}^{\frac{\delta}{2}}} .
$$

Therefore, $\forall \alpha>0, \forall \delta \in(0, \alpha), \forall E \subset[0,1)$ we have

$$
H^{\alpha}(E) \leq H^{\alpha}(E, \Phi) \leq K(\alpha, \delta) H^{\alpha-\delta}(E) .
$$

Hence, $\operatorname{dim}_{H}(E, \Phi) \leq \operatorname{dim}_{H}(E)+\delta, \forall \delta \in(0, \alpha)$, which proves the equality $\operatorname{dim}_{H}(E, \Phi)=$ $\operatorname{dim}_{H}(E)$.

Corollary 1. If $\varlimsup_{n \rightarrow \infty} \frac{q_{n+1}}{q_{n}}<1$, then $\Phi$ is faithful.

Remark 1. From the latter theorem it follows that $\Phi$ is faithful for the case where the series $\sum_{i=1}^{\infty} q_{i}$ converges rather quickly in the sense of (2).

The following rather unexpected Theorem shows that the fine covering system generated by the $Q_{\infty}$-expansion is not necessarily faithful, which shows a new phenomenon related to infinite IFS.

Theorem 2. If there exist constants $m_{0}>1, A>0$ and $B>0$ such that

$$
\frac{A}{i^{m_{0}}} \leq q_{i} \leq \frac{B}{i^{m_{0}}}, \forall i \in \mathbb{N}
$$

then the fine covering system generated by the $Q_{\infty}$-expansion is non-faithful.

Proof. Let us construct a set whose Hausdorff dimension is equal to zero, but the corresponding value of the Hausdorff dimension w.r.t. the fine covering system $\Phi$ is at least $\frac{1}{m_{0}}$.

To this end we shall investigate properties of the Cantor-like set

$$
C\left[Q_{\infty},\left\{V_{k}\right\}\right]=\left\{x: \quad x=\Delta_{\alpha_{1}(x) \ldots \alpha_{k}(x) \ldots}, \alpha_{k}(x) \in V_{k}\right\},
$$

where

$$
V_{k}=\left\{i: \quad i \in \mathbb{N}, l_{2 k} \leq i \leq l_{2 k+1}\right\}
$$

and the sequences $\left\{l_{2 k}\right\},\left\{l_{2 k+1}\right\},\left\{M_{k}\right\}$ are defined recursively as follows:

$$
\begin{gathered}
M_{0}:=1, \quad l_{2}=2 M_{0}=2, \quad l_{3}=\left(l_{2}+1\right)^{2}=9 ; \\
l_{2 k}=\left(2^{k} M_{k-1}\right)^{k}, l_{2 k+1}=\left(l_{2 k}+1\right)^{2}, M_{k-1}=l_{3} \cdot l_{5} \cdot \ldots \cdot l_{2 k-1}, \forall k \in \mathbb{N} .
\end{gathered}
$$

Firstly, let us prove that $\operatorname{dim}_{H}\left(C\left[Q_{\infty},\left\{V_{k}\right\}\right]\right)=0$. From the construction of the set $C\left[Q_{\infty},\left\{V_{k}\right\}\right]$ it follows that for any $k \in \mathbb{N}$ this set can be covered by $M_{k}$ cylinders of rank $k$.

On the other hand, this set can be covered by $M_{k-1}$ intervals $\nabla_{\alpha_{1} \ldots \alpha_{k-1}}$, which are unions of cylinders of rank $k$, i.e.,

$$
\nabla_{\alpha_{1} \ldots \alpha_{k-1}}=\bigcup_{i=l_{2 k}}^{l_{2 k+1}} \Delta_{\alpha_{1} \ldots \alpha_{k-1} i}
$$

It is not hard to see that

$$
\left|\nabla_{\alpha_{1} \ldots \alpha_{k-1}}\right| \leq \sum_{i=l_{2 k}}^{l_{2 k+1}} \frac{B}{i^{m_{0}}} \leq \int_{l_{2 k}-1}^{l_{2 k+1}} \frac{B}{x^{m_{0}}} d x \leq \frac{2^{m_{0}-1} B}{l_{2 k}^{m_{0}-1}} .
$$


Therefore, for any positive $\alpha$ the $\alpha$-volume of the latter covering of the set $C\left[Q_{\infty},\left\{V_{k}\right\}\right]$ does not exceed the value

$$
\frac{M_{k-1} 2^{\alpha\left(m_{0}-1\right)} B^{\alpha}}{\left(2^{k} M_{k-1}\right)^{\alpha k\left(m_{0}-1\right)}}
$$

which tends to zero as $k$ tends to infinity.

So,

$$
\operatorname{dim}_{H}\left(C\left[Q_{\infty},\left\{V_{k}\right\}\right]\right)=0 .
$$

Now we show that $\operatorname{dim}_{H}\left(C\left[Q_{\infty},\left\{V_{k}\right\}\right], \Phi\right)>0$.

Let $\xi$ be the random variable with independent $Q_{\infty}$-digits, which is uniformly distributed on the set $C\left[Q_{\infty},\left\{V_{k}\right\}\right]$, i. e., $\xi$ is of the form

$$
\xi=\Delta_{\xi_{1} \ldots \xi_{k} \ldots},
$$

where $\xi_{k}$ are independent random variables taking values

$$
l_{2 k}, \quad l_{2 k}+1, \ldots, l_{2 k+1}
$$

with probabilities

$$
\frac{1}{\gamma_{k}} \cdot q_{l_{2 k}}, \frac{1}{\gamma_{k}} \cdot q_{l_{2 k}+1}, \ldots, \frac{1}{\gamma_{k}} \cdot q_{l_{2 k+1}}
$$

correspondingly. The normalizing constants $\gamma_{k}$ are determined by

$$
\sum_{i=l_{2 k}}^{l_{2 k+1}} \frac{1}{\gamma_{k}} q_{i}=1 .
$$

Then, taking into account assumptions (41), we get

$$
\begin{gathered}
\gamma_{k} \geq A \cdot \sum_{i=l_{2 k}}^{l_{2 k+1}} \frac{1}{i^{m_{0}}}=A \cdot\left(\frac{1}{l_{2 k}^{m_{0}}}+\sum_{i=l_{2 k}+1}^{l_{2 k+1}} \frac{1}{i^{m_{0}}}\right) \geq \\
\geq A \cdot\left(\frac{1}{l_{2 k}^{m_{0}}}+\int_{l_{2 k}+1}^{l_{2 k+1}+1} \frac{1}{x^{m_{0}}} d x\right) \geq \frac{A}{\left(m_{0}-1\right) \cdot\left(l_{2 k}+1\right)^{m_{0}-1}} .
\end{gathered}
$$

Let $\mu_{\xi}$ be the probability measure of $\xi$. Then

$$
\mu_{\xi}\left(\Delta_{\alpha_{1} \ldots \alpha_{k}}\right)=\frac{1}{\gamma_{1}} q_{\alpha_{1}} \cdot \ldots \cdot \frac{1}{\gamma_{k}} q_{\alpha_{k}} \leq \frac{1}{\gamma_{k}} \cdot q_{\alpha_{k}} \leq \frac{D \cdot l_{2 k}^{m_{0}-1}}{\alpha_{k}^{m_{0}}},
$$

where $D=\frac{B\left(m_{0}-1\right) 2^{m_{0}-1}}{A}$.

On the other hand we have

$$
\left|\Delta_{\alpha_{1} \alpha_{2} \ldots \alpha_{k}}\right| \geq \frac{A^{k}}{M_{k-1}^{m_{0}}} \cdot \frac{1}{\alpha_{k}^{m_{0}}} .
$$

So, for any $x \in C\left[Q_{\infty},\left\{V_{k}\right\}\right]$ and for any $\alpha \in\left(0, \frac{1}{m_{0}}\right)$

$$
\begin{aligned}
\frac{\mu_{\xi}\left(\Delta_{\alpha_{1}(x) \alpha_{2}(x) \ldots \alpha_{k}(x)}\right)}{\left|\Delta_{\alpha_{1}(x) \alpha_{2}(x) \ldots \alpha_{k}(x)}\right|^{\alpha}} & \leq \frac{D \cdot l_{2 k}^{m_{0}-1}}{\alpha_{k}^{m_{0}}}:\left(\frac{A^{k}}{M_{k-1}^{m_{0}} \alpha_{k}^{m_{0}}}\right)^{\alpha} \leq \\
& \leq \frac{D}{M_{k-1}^{k\left(1-\alpha m_{0}\right)-\alpha m_{0}} \cdot\left(A^{\alpha} 2^{k\left(1-\alpha m_{0}\right)}\right)^{k}} .
\end{aligned}
$$

So,

$$
\lim _{k \rightarrow \infty} \frac{\mu_{\xi}\left(\Delta_{\alpha_{1}(x) \alpha_{2}(x) \ldots \alpha_{k}(x)}\right)}{\left|\Delta_{\alpha_{1}(x) \alpha_{2}(x) \ldots \alpha_{k}(x)}\right|^{\alpha}}=0
$$


for all $\alpha \in\left(0, \frac{1}{m_{0}}\right)$ and $x \in C\left[Q_{\infty},\left\{V_{k}\right\}\right]$, and, therefore,

$$
\operatorname{dim}_{H}\left(C\left[Q_{\infty},\left\{V_{k}\right\}\right], \Phi\right) \geq \frac{1}{m_{0}} \neq 0=\operatorname{dim}_{H}\left(C\left[Q_{\infty},\left\{V_{k}\right\}\right]\right),
$$

which proves the theorem.

Remark 2. The proof of the latter theorem is based on the method which was invented by Yuval Peres (see [38 for details) to prove the non-faithfulness of the family of continued fraction cylinders.

Remark 3. One can prove that $\operatorname{dim}_{H}\left(C\left[Q_{\infty},\left\{V_{k}\right\}\right], \Phi\right)=\frac{1}{m_{0}}$.

Corollary 2. If $q_{i}=\frac{1}{P_{m_{0}}(i)}$ for all $i$ which are large enough and for a polynomial $P_{m_{0}}(x)$ of a degree $m_{0}>1$, then the corresponding fine covering family generated by this $Q_{\infty}$ expansion is non-faithful.

Corollary 3. The family of cylinders generated by the Lüroth expansion [19, 45] is nonfaithful.

Open problem. The problem of finding necessary and sufficient conditions for the faithfulness resp. non-faithfulness of a family of cylinders of a given $Q_{\infty}$-expansion is still open.

\section{Superfractality of THE SeT of $Q_{\infty}$-NON-NORMAL NUMBers}

The main aim of this section is to prove the superfractality of the set of $Q_{\infty}$-essentially non-normal numbers without any additional restrictions on the stochastic vector $Q_{\infty}$.

Theorem 3. The set $L\left(Q_{\infty}\right)$ of $Q_{\infty}$-essentially non-normal numbers is of full Hausdorff dimension.

Proof. The main idea of the proof is rather clear: to construct a countable family of subsets from $L\left(Q_{\infty}\right)$ whose Hausdorff dimension can be arbitrarily close to unity. Possible infinite entropy of the stochastic vector $Q_{\infty}$, possible non-faithfulness of the family $\Phi\left(Q_{\infty}\right)$ and the absence of general formulae for the calculation of the Hausdorff dimension for probability measures with independent $Q_{\infty}$-symbols (this is still an open problem (see, e.g., [33])) do not allow us to apply methods from [2, 9] to construct such a family. Methods from [34, 35] are also not applicable to solve the problem because of the absence of 'divergent points techniques' for the measures generated by infinite IFS.

To overcome the above problems, for any stochastic vector $Q_{\infty}$ we shall construct a two-parametric family of subsets $T_{s, l}=T_{s, l}\left(Q_{\infty}\right)$ with a desired properties and apply the developed probabilistic and Hausdorff dimension techniques to estimate $\operatorname{dim}_{H}\left(T_{s, l}\right)$.

So, let $s$ and $l>2$ be fixed positive integers. For a given stochastic vector $Q_{\infty}=$ $\left(q_{0}, q_{1}, \ldots, q_{i}, \ldots\right)$ let $m_{0}:=1$ and $m_{i}:=\left[\ln ^{2} q_{i}\right] \cdot 2^{i}, \forall i \in \mathbb{N}$, and let $T_{s, l}$ be the set of real numbers whose $Q_{\infty}$-symbols can be separated into groups such that the $k$-th group is of the following structure

$$
\alpha_{k, 1} \ldots \alpha_{k, s R_{k}} \underbrace{0 \ldots 0}_{R_{k}} \underbrace{1 \ldots 1}_{\frac{R_{k}}{m_{1}}} \cdots \underbrace{i \ldots i}_{\frac{R_{k}}{m_{i}}} \cdots \underbrace{(k-2) \ldots(k-2)}_{\frac{R_{k}}{m_{k-2}}} \underbrace{(k-1) \ldots(k-1)}_{\frac{R_{k} m_{k}}{m_{k-1}\left(m_{k}-1\right)}},
$$

where $R_{1}:=m_{1}$, and $R_{k}:=m_{1} m_{2} \cdot \ldots \cdot m_{k-1}\left(m_{k}-1\right), \forall k \geq 2$ and symbols $\alpha_{k, 1}, \alpha_{k, 2}, \ldots$, $\alpha_{k, s R_{k}}$ can be chosen independently from the set $\{0,1, \ldots, l-1\}$.

Let us denote by $\operatorname{Fix}(j)$ the set of numbers of positions of the fixed digit ' $j$ ' in the $Q_{\infty}$-expansion of $x \in T_{s, l}$. 
Let

$$
\text { Fix }=\bigcup_{j=0}^{\infty} \operatorname{Fix}(j) ; \quad \text { Flex }=\mathbb{N} \backslash \text { Fix. }
$$

Then the set $T_{s, l}$ can be defined by

$$
\begin{aligned}
T_{s, l}=\{x: \quad & x=\Delta_{\alpha_{1}(x) \alpha_{2}(x) \ldots \alpha_{n}(x) \ldots ;} ; \\
& \alpha_{n}(x)=j \text { for all } n \in \operatorname{Fix}(j), j \in \mathbb{N}_{0} ; \\
& \left.\alpha_{n}(x) \in\{0,1, \ldots, l-1\} \text { for all } n \in \text { Flex }\right\} .
\end{aligned}
$$

Firstly let us show that $T_{s, l} \subset L\left(Q_{\infty}\right)$. To this end we must prove that for any $x \in T_{s, l}$ and for any digit $i \in \mathbb{N}_{0}$ the limit $\lim _{n \rightarrow \infty} \frac{N_{i}(x, n)}{n}$ does not exist. Since the proof is very similar for all digits we shall explain it only for the digit 0 . Let $n_{k}^{0}$ be the number of the position at which the series of fixed zeros of the $k$-th group is ended. From the construction of the set $T_{s, l}$ it follows that

$$
n_{k}^{0}=(s+1) \prod_{i=1}^{k} m_{i}+\sum_{j=1}^{k-2}\left(\frac{\prod_{i=1}^{k-1} m_{i}}{m_{j}}\right) .
$$

Let $n_{k}^{1}$ be the number of the position at which the series of fixed ones of the $k$-th group is ended, i. e., $n_{k}^{1}=n_{k}^{0}+\frac{R_{k}}{m_{1}}$. Then

$$
N_{0}\left(x, n_{k}^{0}\right)=\prod_{i=1}^{k} m_{i}+\tau_{0}\left(x, n_{k}^{0}\right),
$$

where $\tau_{0}\left(x, n_{k}^{0}\right)$ is the number of zeros among the first non-fixed digits until the position $n_{k}^{0}$.

It is clear that $N_{0}\left(x, n_{k}^{0}\right)=N_{0}\left(x, n_{k}^{1}\right)$ and

$$
\begin{aligned}
& \frac{N_{0}\left(x, n_{k}^{0}\right)}{n_{k}^{0}}=\frac{1+\tau_{0}\left(x, n_{k}^{0}\right)\left(\prod_{i=1}^{k} m_{i}\right)^{-1}}{s+1+\frac{1}{m_{k}} \sum_{i=1}^{k-2} \frac{1}{m_{i}}} ; \\
& \frac{N_{0}\left(x, n_{k}^{1}\right)}{n_{k}^{1}}=\frac{1+\tau_{0}\left(x, n_{k}^{0}\right)\left(\prod_{i=1}^{k} m_{i}\right)^{-1}}{s+1+\frac{1}{m_{1}}+\frac{1}{m_{k}} \sum_{i=2}^{k-2} \frac{1}{m_{i}}} .
\end{aligned}
$$

If the limit $\lim _{k \rightarrow \infty} \tau_{0}\left(x, n_{k}^{0}\right)\left(\prod_{i=1}^{k} m_{i}\right)^{-1}$ does not exist, then the limit $\lim _{k \rightarrow \infty} \frac{N_{0}(x, k)}{k}$ also does not exist.

If the limit $\lim _{k \rightarrow \infty} \tau_{0}\left(x, n_{k}^{0}\right)\left(\prod_{i=1}^{k} m_{i}\right)^{-1}=a(x)$ exists, then

$$
\lim _{k \rightarrow \infty} \frac{N_{0}\left(x, n_{k}^{0}\right)}{n_{k}^{0}}=\frac{1+a(x)}{s+1} \text { and } \lim _{k \rightarrow \infty} \frac{N_{0}\left(x, n_{k}^{1}\right)}{n_{k}^{1}}=\frac{1+a(x)}{s+1+\frac{1}{m_{1}}} .
$$

Therefore, for any $x \in T_{s, l}$ the digit ' 0 ' does not have the frequency in the $Q_{\infty}$-expansion of $x$.

Theorem 2 shows that the family $\Phi\left(Q_{\infty}\right)$ could be non-faithful for the Hausdorff dimension calculation on the unit interval. Nevertheless we show that for any stochastic vector 
$Q_{\infty}$ the family $\Phi$ of all $Q_{\infty}$-cylinders is admissible for the Hausdorff dimension calculation of $T_{s, l}$, i. e.,

$$
\operatorname{dim}_{H}\left(T_{s, l}\right)=\operatorname{dim}_{H}\left(T_{s, l}, \Phi\right) .
$$

To this end let us consider an arbitrary covering of $T_{s, l}$ by closed intervals $E_{j}=\left[a_{j}, b_{j}\right]$. Let $I_{j}:=T_{s, l} \cap E_{j}$.

Let $\Delta^{j}$ be the cylinder of minimal length among all cylinders containing the set $I_{j}$, and let $k_{j}$ be the rank of $\Delta^{j}$. Then $\left(k_{j}+1\right) \in$ Flex.

Let

$$
\Delta^{j}=\Delta_{\alpha_{1} \ldots \alpha_{k_{j}}}=\bigcup_{i=0}^{\infty} \Delta_{\alpha_{1} \ldots \alpha_{k_{j}}} .
$$

Let $c_{j}:=\inf I_{j}, d_{j}:=\sup I_{j}$, then $c_{j} \in \Delta_{\alpha_{1} \ldots \alpha_{k_{j}} 0}$. From the construction of the set $T_{s, l}$ it follows that $T_{s, l} \cap \Delta_{\alpha_{1} \ldots \alpha_{k_{j}} i}=\emptyset, \forall i \geq l$ and $T_{s, l} \cap \Delta_{\alpha_{1} \ldots \alpha_{k_{j}}(l-1)} \neq \emptyset$. Since $d_{j}$ is the supremum of $I_{j}$, we conclude that $d_{j} \in \Delta_{\alpha_{1} \ldots \alpha_{k_{j}}(l-1)}$. Hence, $\Delta_{\alpha_{1} \ldots \alpha_{k_{j}} 1} \subset\left[c_{j}, d_{j}\right]$, and, therefore

$$
\left|\left(c_{j}, d_{j}\right)\right|>\left|\Delta_{\alpha_{1} \ldots \alpha_{k_{j}} 1}\right|=q_{1} \cdot\left|\Delta_{\alpha_{1} \ldots \alpha_{k_{j}}}\right| .
$$

So, $\left|\Delta^{j}\right|<\frac{1}{q_{1}}\left|E_{j}\right|$. Thus, for any closed interval $E_{j}$ we can cover the set $T_{s, l} \cap E_{j}$ by one cylinder of length not larger than $\frac{1}{q_{1}}\left|E_{j}\right|$.

Therefore,

$$
H^{\alpha}\left(T_{s, l}\right) \leq H^{\alpha}\left(T_{s, l}, \Phi\right) \leq \frac{1}{q_{1}^{\alpha}} H^{\alpha}\left(T_{s, l}\right)
$$

for any $\alpha \in(0,1]$, and, hence, $\operatorname{dim}_{H}\left(T_{s, l}, \Phi\right)=\operatorname{dim}_{H}\left(T_{s, l}\right)$.

Finally, let us estimate the Hausdorff dimension of the set $T_{s, l}$. Our purpose is to prove that the Hausdorff dimension of the set $T_{s, l}$ is close to 1 for large enough $s$ and $l$. To this end we construct a special singularly continuous probability measure such that the set $T_{s, l}$ is the topological support of the measure.

Let $n_{k}$ be the number of the position at which the $k$-th group is ended.

The number of non-fixed digits $j \in$ Flex among the first $n_{k}$ digits is equal to $s \cdot \prod_{i=1}^{k} m_{i}$. The number of a fixed digit $j(j \in\{0,1, \ldots, k-1\})$ among the first $n_{k}$ digits is equal to $\frac{\prod_{i=1}^{k} m_{i}}{m_{j}}$.

Let $\xi(l)$ be a random variable with independent $Q_{\infty}$-digits $\xi_{k}(l)$ defined by

$$
\xi(l)=\Delta_{\xi_{1}(l) \xi_{2}(l) \ldots \xi_{k}(l) \ldots},
$$

where $\xi_{k}(l)$ has the following distributions:

- if $k \in \operatorname{Fix}(j)$, then

- if $k \in$ Flex, then

$$
\begin{array}{c|c}
\xi_{k}(l) & j \\
\hline & p_{j k}=1
\end{array}, j \in \mathbb{N}_{0} ;
$$

\begin{tabular}{c|c|c|c|c}
$\xi_{k}(l)$ & 0 & 1 & $\ldots$ & $l-1$ \\
\hline & $p_{0 k}=\frac{q_{0}}{S_{l}}$ & $p_{1 k}=\frac{q_{1}}{S_{l}}$ & $\ldots$ & $p_{(l-1) k}=\frac{q_{l-1}}{S_{l}}$
\end{tabular}

where $S_{l}:=\sum_{i=0}^{l-1} q_{i}$ 
Let $\mu_{\xi(l)}$ be the above defined probability distribution of the corresponding random variable $\xi(l)$ with independent $Q_{\infty}$-digits. It is clear that $T_{s, l}$ is the topological support of the measure $\mu_{\xi(l)}$. So,

$$
\operatorname{dim}_{H}\left(T_{s, l}\right)=\operatorname{dim}_{H}\left(T_{s, l}, \Phi\right) \geq \operatorname{dim}_{H}\left(\mu_{\xi(l)}, \Phi\right) .
$$

Fine fractal properties of probability measures with independent $Q_{\infty}$-symbols were studied in [33]. In particular, it has been proven there that under the assumptions

$$
\sum_{k=1}^{\infty} \frac{\sum_{i=0}^{\infty} p_{i k} \ln ^{2} p_{i k}}{k^{2}}<\infty \text { and } \sum_{k=1}^{\infty} \frac{\sum_{i=0}^{\infty} p_{i k} \ln ^{2} q_{i}}{k^{2}}<\infty
$$

the Hausdorff dimension of the measure with respect to $\Phi$ can be calculated as follows

$$
\operatorname{dim}_{H}\left(\mu_{\xi(l)}, \Phi\right)=\varliminf_{n \rightarrow \infty} \frac{\sum_{k=1}^{n} h_{k}}{\sum_{k=1}^{n} b_{k}},
$$

where

$$
h_{k}:=-\sum_{i=0}^{\infty} p_{i k} \ln p_{i k}, \quad b_{k}:=-\sum_{i=0}^{\infty} p_{i k} \ln q_{i} .
$$

In our case $\sum_{k=1}^{\infty} \frac{\sum_{i=0}^{\infty} p_{i k} \ln ^{2} p_{i k}}{k^{2}}<\infty$, because

$$
\sum_{i=0}^{\infty} p_{i k} \ln ^{2} p_{i k}= \begin{cases}0, & \text { if } k \in \text { Fix } \\ \sum_{i=0}^{l-1} \frac{q_{i}}{S_{l}} \ln ^{2} \frac{q_{i}}{S_{l}}, & \text { if } k \in \text { Flex }\end{cases}
$$

Let us show that $\sum_{k=1}^{\infty} \frac{\sum_{i=0}^{\infty} p_{i k} \ln ^{2} q_{i}}{k^{2}}<\infty$ as well.

Since

$$
\sum_{i=0}^{\infty} p_{i k} \ln ^{2} q_{i}= \begin{cases}\ln ^{2} q_{j}, & \text { if } k \in \operatorname{Fix}(j), \quad j \in \mathbb{N}_{0} \\ \sum_{i=0}^{l-1} \frac{q_{i}}{S_{l}} \ln ^{2} q_{i}, & \text { if } k \in \text { Flex },\end{cases}
$$

it is enough to prove the convergence of the series $\sum_{j=0}^{\infty}\left(\sum_{i \in \operatorname{Fix}(j)} \frac{\ln ^{2} q_{j}}{i^{2}}\right)$.

From the construction of $T_{s, l}$ it follows that $m_{j}$ is less than the minimal element of the set $\operatorname{Fix}(j)$. Then

$$
\sum_{j=0}^{\infty}\left(\sum_{i \in \operatorname{Fix}(j)} \frac{\ln ^{2} q_{j}}{i^{2}}\right)<\sum_{j=0}^{\infty}\left(\sum_{i=m_{j}+1}^{\infty} \frac{\ln ^{2} q_{j}}{i(i-1)}\right)=\sum_{j=0}^{\infty} \frac{\ln ^{2} q_{j}}{m_{j}}<\infty .
$$

Since $h_{k} \leq b_{k}$, it is not hard to check that $\varliminf_{n \rightarrow \infty} \frac{\sum_{i=1}^{n} h_{i}}{\sum_{i=1}^{n} b_{i}}=\varliminf_{k \rightarrow \infty} \frac{\sum_{i=1}^{n_{k}} h_{i}}{\sum_{i=1}^{n_{k}} b_{i}}$. 
Let $\mathbf{h}_{l}:=\sum_{i=0}^{l-1} \frac{q_{i}}{S_{l}} \ln \frac{S_{l}}{q_{i}}, \quad \mathbf{b}_{l}:=\sum_{i=0}^{l-1} \frac{q_{i}}{S_{l}} \ln \frac{1}{q_{i}}$. Then

$$
\sum_{i=1}^{n_{k}} h_{i}=s \mathbf{h}_{l} \prod_{i=1}^{k} m_{i} \quad \text { and } \quad \sum_{i=1}^{n_{k}} b_{i}=s \mathbf{b}_{l} \prod_{i=1}^{k} m_{i}+\prod_{i=1}^{k} m_{i}\left(\sum_{j=0}^{k-1} \frac{1}{m_{j}} \ln \frac{1}{q_{j}}\right) .
$$

So,

$$
\frac{\sum_{i=1}^{n_{k}} h_{i}}{\sum_{i=1}^{n_{k}} b_{i}}=\frac{s \mathbf{h}_{l}}{s \mathbf{b}_{l}+\ln \frac{1}{q_{0}}+\sum_{i=1}^{k-1} \frac{1}{m_{i}} \ln \frac{1}{q_{i}}}=\frac{s \mathbf{h}_{l}}{s \mathbf{b}_{l}+\ln \frac{1}{q_{0}}+\sum_{i=1}^{k-1} \frac{1}{\left[\ln ^{2} \frac{1}{q_{i}}\right] 2^{i}} \ln \frac{1}{q_{i}}}
$$

Let $K:=\lim _{k \rightarrow \infty}\left(\ln \frac{1}{q_{0}}+\sum_{i=1}^{k-1} \frac{\ln \frac{1}{q_{i}}}{\left[\ln ^{2} \frac{1}{q_{i}}\right] 2^{i}}\right)<\infty$. Then $\lim _{k \rightarrow \infty} \frac{\sum_{i=1}^{n_{k}} h_{i}}{\sum_{i=1}^{n_{k}} b_{i}}=\frac{s \mathbf{h}_{l}}{s \mathbf{b}_{l}+K}$.

So, taking into account (5), we get $\operatorname{dim}_{H} T_{s, l} \geq \frac{s \mathbf{h}_{l}}{s \mathbf{b}_{l}+K}$.

Since $S_{l} \rightarrow 1(l \rightarrow \infty)$, we get

$$
\operatorname{dim}_{H}\left(L\left(Q_{\infty}\right)\right) \geq \sup _{s, l} \operatorname{dim}_{H} T_{s, l}=1,
$$

which proves the theorem.

\section{Acknowledgment}

This work was partly supported by SFB-701 "Spectral Structures and Topological Methods in Mathematics" (Bielefeld University), STREVCOM FP-7-IRSES 612669 project and by the Alexander von Humboldt Foundation. The authors would like to express their gratitude to Prof. Yuval Peres (Microsoft Research) for fruitful discussions and valuable remarks on problems related to the non-faithfulness of coverings.

\section{REFERENCES}

[1] S. Albeverio, Yu. Kondratiev, Yu. Kozitsky, M. Röckner, The statistical mechanics of quantum lattice systems. A path integral approach. EMS Tracts in Mathematics, 8. European Mathematical Society (EMS), Zürich, 2009.

[2] S. Albeverio, Yu. Kondratiev, R. Nikiforov, G. Torbin, On fractal properties of non-normal numbers with respect to Rényi $f$-expansions generated by piecewise linear functions, Bull. Sci. Math., 138 (2014), no. 3, $440-455$.

[3] S. Albeverio, G. Torbin, Fractal properties of singularly continuous probability distributions with independent $Q^{*}$-digits, Bull. Sci. Math., 129 (2005), no. 4, 356 - 367.

[4] S.Albeverio, M.Pratsiovytyi, G.Torbin, Singular probability distributions and fractal properties of sets of real numbers defined by the asymptotic frequencies of their s-adic digits, Ukrainian Mathematical Journal, 57(2005), 1361-1370.

[5] S.Albeverio, G.Torbin, On fine fractal properties of generalized infinite Bernoulli convolutions, Bulletin des Sciences Mathematiques, 132(2008), P. 711-727.

[6] S. Albeverio, O. Baranovskyi, M. Pratsiovytyi, G. Torbin, The Ostrogradsky series and related Cantor-like sets, Acta Arithm., 130(2007), no. 3. - P. 215 - 230.

[7] S.Albeverio, G.Torbin. Image measures of infinite product measures and generalized Bernoulli convolutions. SFB 611 Preprint and Transactions of the National Pedagogical University (Phys.-Math. Sci.), 5(2004), $228-241$.

[8] S. Albeverio, V. Koshmanenko, M. Pratsiovytyi, G. Torbin , On fine structure of singularly continuous probability measures and random variables with independent $\tilde{Q}$-symbols. Methods of Functional Analysis and Topology. 2011. no. 1. 97-111. 
[9] S. Albeverio, M. Pratsiovytyi, G. Torbin, Topological and fractal properties of real numbers which are not normal, Bull. Sci. Math., 129 (2005), no. 8, 615-630.

[10] I. S. Baek, L. Olsen, N. Snigireva, Divergence points of self-similar measures and packing dimension, Adv. Math., 214 (2007), no. 1, 267 - 287.

[11] L. Barreira, G. Iommi, Frequency of digits in the Lüroth expansion, J. Number Theory, 129 (2009), no. $6,1479-1490$.

[12] L. Barreira, B. Saussol, J. Schmeling, Distribution of frequencies of digits via multifractal analysis, J. Number Theory, 97 (2002), no. 2, $410-438$.

[13] M. P. Bernardi, C. Bondioli, On some dimension problems for self-affine fractals, Z. Anal. Anwendungen, 18 (1999), no. 3, $733-751$.

[14] A. Besicovitch, On the sum of digits of real numbers represented in the dyadic system, Math. Ann., 110 (1935), no. 1, $321-330$.

[15] P. Billingsley, Ergodic theory and information, John Willey and Sons, New York, 1965.

[16] A. Bisbas, N. Snigireva, Divergence points and normal numbers, Monatsh. Math., 166 (2012), no. 3$4,341-356$.

[17] R. Bowen, Equilibrium states and the ergodic theory of Anosov diffeomorphisms, Lecture Notes in Mathematics, Vol. 470. Springer-Verlag, Berlin-New York, 1975.

[18] C. D. Cutler, A note on equivalent interval covering systems for Hausdorff dimension on $\mathbb{R}$, Internat. J. Math. and Math. Sci., 4 (1988), Vol. II, $643-650$.

[19] K. Dajani, C. Kraaikamp, Ergodic theory of numbers, Mathematical Association of America, Washington, 2002.

[20] H. Eggleston, The fractional dimension of a set defined by decimal properties, Quart. J. Math., Oxford Ser. 20 (1949), $31-36$.

[21] C. I. Everett, Representations for real numbers, Bull. Amer. Math. Soc., 52 (1946), 861 - 869.

[22] K. J. Falconer, Techniques in fractal geometry, Willey, Chichester, 1997.

[23] A. H. Fan, L. M. Liao, J. H. Ma, B. W. Wang, Dimension of Besicovitch-Eggleston sets in countable symbolic space, Nonlinearity, 23 (2010), no. 5, 1185 - 1197.

[24] A. H. Fan, L. M. Liao, J. H. Ma, On the frequency of partial quotients of regular continued fractions, Math. Proc. Cambridge Philos. Soc., 148 (2010), no. 1, 179 - 192.

[25] I. J. Good, The fractional dimensional theory of continued fractions, Proc. Cambridge Phil. Soc., 37(1941), $199-228$.

[26] D. Hensley, The Hausdorff dimensions of some continued fraction Cantor sets, J. Number Theory, 33(1989), no. 2, $182-198$.

[27] D. Hensley, A polynomial time algorithm for the Hausdorff dimension of continued fraction Cantor sets, J. Number Theory, 58(1996), no. 1, $9-45$.

[28] O. Jenkinson, M. Pollicott, Computing the dimension of dynamically defined sets: $E_{2}$ and bounded continued fractions Ergod. Th. and Dynam. Sys., 21(2001), no. 5, $1429-1445$.

[29] A. Ya. Khintchine, Continued fractions, P. Noordhoff, Ltd., Groningen, 1963.

[30] T. Łuczak, On the fractional dimension of sets of continued fractions, Mathematika, 44 (1997), no. 1, $50-53$.

[31] R. D. Mauldin, M. Urbański, Dimensions and measures in infinite iterated function systems, Proc. London Math. Soc. (3), 73 (1996), no. 1, $105-154$.

[32] R. Nikiforov, G. Torbin, Ergodic properties of the $Q_{\infty}$-expansion and fractal properties of probability measures with independent $Q_{\infty}$-digits, Transactions of Dragomanov National Pedagogical University. Series 1: Phys.-Math. Sciences, 9 (2008), $150-174$.

[33] R. Nikiforov, G. Torbin, Fractal properties of random variables with independent $Q_{\infty}$-digits, Theory Probab. Math. Stat., 86 (2013), 169 - 182.

[34] L. Olsen, Applications of multifractal divergence points to sets of numbers defined by their $N$-adic expansion, Math. Proc. Cambridge Philos. Soc., 136 (2004), no. 1, 139 - 165.

[35] L. Olsen, Applications of multifractal divergence points to some sets of $d$-tuples of numbers defined by their $N$-adic expansion. Bull.Sci.Math., 128 (2004), no. 4, $265-289$.

[36] L. Olsen, Hausdorff and packing dimensions of non-normal tuples of numbers: non-linearity and divergence points, Bull. Sci. Math., 134 (2010), no. 1, $64-96$.

[37] L. Olsen, S. Winter, Normal and non-normal points of self-similar sets and divergence points of self-similar measures, J. London Math. Soc. (2), 67 (2003), no. 1, 103 - 122.

[38] Yu. Peres, G. Torbin, Continued fractions and dimensional gaps, in preparation. 
[39] Y. Pesin, Dimension theory in dynamical systems. Contemporary views and applications, Chicago Lectures in Mathematics. University of Chicago Press, Chicago, (1997).

[40] M. Pratsiovytyi, G. Torbin, Superfractality of the set of numbers having no frequency of $n$-adic digits, and fractal probability distributions, Ukrainian Math. J., 47 (1995), no. 7, $971-975$. S

[41] M. Pratsiovytyi, G. Torbin, On analytic (symbolic) representation of one-dimensional continuous transformations preserving the Hausdorff-Besicovitch dimension, Transactions of Dragomanov National Pedagogical University. Series 1: Phys.-Math. Sciences, 4 (2003), 207 - 205.

[42] A. Rényi, Representations for real numbers and their ergodic properties, Acta Math. Sci. Hungar., 8 (1957), $477-493$.

[43] C. A. Rogers, Hausdorff measures, Cambridge Univ. Press, London, 1970.

[44] D. Ruelle, Thermodynamic formalism. The mathematical structures of equilibrium statistical mechanics. Cambridge University Press, Cambridge, 2004.

[45] F. Schweiger, Ergodic theory of fibred systems and metric number theory, Oxford University Press, New York, 1995.

[46] G. Torbin, Fractal properties of the distributions of random variables with independent Q-symbols, Transactions of the National Pedagogical University (Phys.-Math. Sci.), 3(2002), 241-252.

[47] G. Torbin. Multifractal analysis of singularly continuous probability measures. Ukrainian Math. J. 57 (2005), no. 5, 837-857.

[48] G. Torbin, Probability distributions with independent Q-symbols and transformations preserving the Hausdorff dimension, Theory of Stochastic Processes,13(2007), 281-293.

[49] A. F. Turbin, M. V. Pratsiovytyi, Fractal sets, functions, distributions, Naukova Dumka, Kiev, 1992.

[50] B. Volkmann, Gewinnmengen, Arch. Math., 10 (1959), 235 - 240. 